\title{
Heterotopic Ossification of the Hip Post Reamed Intramedullary Femoral Nail: A Case Report
}

\author{
Kow RY*, Low CL, Jaya Raj J, VA Jacob Abraham and ESK Mor Japar Khan \\ Hospital Tengku Ampuan Afzan, Malaysia
}

ISSN: 2576-8875

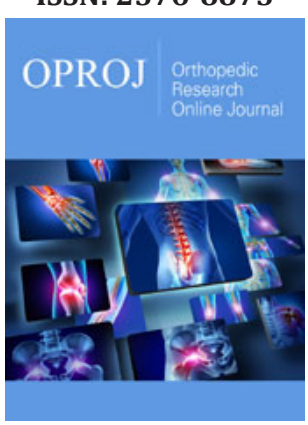

${ }^{* 1}$ Corresponding author: Kow Ren Yi, Hospital Tengku Ampuan Afzan, Malaysia

Submission: 眥 May 07, 2019

Published: 啙June 03, 2019

Volume 5 - Issue 4

How to cite this article: Kow RY, Low CL, Jaya Raj J, VA Jacob A, ESK Mor Japar Khan. Heterotopic Ossification of the Hip Post Reamed Intramedullary Femoral Nail: A Case Report. Ortho Res Online J. 5(4) OPROJ.000619.2019.

DOI: 10.31031/OPROJ.2019.05.000619

Copyright@: Kow Ren Yi, This article is distributed under the terms of the Creative Commons Attribution 4.0 International License, which permits unrestricted use and redistribution provided that the original author and source are credited.

\begin{abstract}
Hetorotopic Ossification (HO) is abnormal formation of bone in the soft tissue. Heterotopic ossification of the hip following reamed interlocking medullary nail is uncommon and it has received little attention from the clinician. We take this opportunity to report a young man who presented with symptomatic heterotopic ossification of the hip eight months post antegrade reamed interlocking medullary nail which failed conservative treatment. Reamed intramedullary nailing and delayed mobilization are the contributing factors in this patient. The pathophysiology of this condition is not fully understood. As a result, there is no consensus in the prevention of heterotopic ossification in such patients. Similarly, the management of this condition also is lacking but resection is the treatment of choice for symptomatic patient.
\end{abstract}

Keywords: Heterotopic ossification; Reamed intramedullary femoral nail; Complication; Femur fracture

\section{Introduction}

Over the years, reamed Intramedullary Nail (IMN) has evolved to become the "standard of care" in the treatment of diaphyseal femur fractures [1-3]. It is a load sharing implant that allows early post-operative weight bearing. It also provides stabilization, easier nursing care and reduces mortality in polytrauma patients. Complications following IMN are prominent and symptomatic hardware, infection, fat embolism, iatrogenic fracture, delayed and nonunion [1]. Heterotopic ossification formation around the hip post IMN is usually overlooked because patients are normally asymptomatic. With this case report, we would like to bring to attention to a common but usually neglected complication of IMN.

\section{Case Report}

Mr VN, a 21-year-old gentleman, sustained open communited fracture of left femur grade IIIa following a motor-vehicle accident on the $7^{\text {th }}$ of March 2013. He has no other injuries. Intravenous antibiotic, wound debridement and skeletal traction $5 \mathrm{~kg}$ was applied. Left femur antegrade reamed intramedullary nail was done 1-week post-trauma once the wound healed. His condition was complicated with surgical site infection at the distal part of the wound postoperative day one. Wound debridement was done, intravenous antibiotic was continued after which he was discharged a week later.

He was discharged with advice for strict non-weight bearing crutches ambulation. He was allowed partial weight bearing 3 months post-IMN insertion after left femur x-ray showed callus formation at the fracture site. He resumed usual full weight bearing on left lower limb 6 months post-IMN insertion.

He started complaining of left hip pain during follow-up clinic visits 8 months post-IMN insertion. The pain was not relieved by analgesia and physiotherapy, and it was aggravated with movement of left hip. There was no bony mass palpable. Range of motion of his left hip was reduced due to the hip pain. X-ray of his left hip showed heterotopic ossification of the left hip which became more radio-opaque with time. He was counselled for excision, but the patient refused. He was referred to physiotherapist for lower limb exercise to reduce the discomfort. The patient claimed that the pain subsided during the subsequent follow up at 3 months, 6 months, 1 year and 3 years respectively. 


\section{Discussion}

Hetorotopic Ossification (HO) is abnormal formation of bone in the soft tissue [4]. HO was first described by Riedel in 1883 and then by Dejerine \& Ceillier in 1918, who found it mainly in soldiers who had spinal cord injury during World War I [4].

HO can be divided into the acquired and hereditary forms [4]. The hereditary form, also known as myositis ossificans is rare [4]. The acquired form of HO may occur following trauma to the musculoskeletal system or injury to spinal cord or central nervous system [4].

HO can occur at any site post-trauma but most commonly affecting the hip, followed by the knee, shoulder, elbow and rarely feet [5]. Literature review shows a high incidence of HO among patients undergoing total hip arthroplasty (30\%) and intramedullary nail of femur fracture $(60 \%)[6,7]$.

The exact etiology of HO is still unknown. However, contributing factors such as hypercalcemia, hip abductor hypoxia, spinal cord injury, prolonged immobilization, mobilization after prolonged immobilization as well as parathyroid and calcitonin imbalance have been reported [5]. The contributing factors are postulated to have increased of prostaglandin E2, which induces periosteal lamellar bone formation. This hypothesis is supported by formation of HO in rats injected with subcutaneous PGE2 [5].

In this patient, prolonged immobilization post trauma causes venous stasis and subsequently arteriovenous shunting around the hip, playing a role in the formation of HO [5]. Besides that, reamed IMN increases the incidence of HO formation compared to nonreamed IMN (35.7\% versus 9.4\%) [2]. In spite of that, reaming debris cannot be blamed as the sole contributing cause as there is $9.4 \%$ of HO formation in non-reamed IMN. There is no literature to support that infection increases the risk of HO formation in this patient.

The signs and symptoms of $\mathrm{HO}$ usually present within 3 to 12 weeks post trauma or spinal cord injury [4]. Initially, the patient may present with pain, fever, swelling and erythema. However, these non-specific symptoms may mimic cellulitis, thrombophlebitis, deep vein thrombosis, osteomyelitis and tumour during the early course of the disease [4]. Up to 20 percent of patients will later developed reduced range of motion and ankylosis of joints [4]. Our patient was asymptomatic until eight months post-ILN and his serial radiograph showed a well demarcated HO formation. Some centers will perform 3-phase bone scan to establish the metabolic activity of the HO.

\section{Prevention}

Studies show that incidence of HO in non-reamed IMN is lower compared to reamed IMN [2]. However, the risks of implant failure, delayed and malunion associated with non-reamed IMN is against the use of non-reamed IMN [3].

Hip abductor ischemia is a known cause of HO [2]. Hence, diligent soft tissue care should be provided during the nailing procedure.
Rehabilitation: Studies in animal show that forcible manipulation induces $\mathrm{HO}$ but there is limited evidence in human studies [5]. Current practise allocates partial weight bearing in patients with callus formation and full weight bearing in patients with hard callus formation. Passive range of motion exercises are recommended to prevent the complication of $\mathrm{HO}$ such as peripheral nerve entrapment, pressure ulcers and functional impairment if joint ankylosis developed [4]. Aggressive joint manipulation has been suggested but it can further worsen the existing HO [4].

Non-Steriodal Anti-Inflammatory Drugs (NSAIDs): Prophylactic NSAIDs (indomethacin and celecoxib) given in patients post total hip arthroplasty has been shown to reduce the risk of HO [5]. However, it is not applicable in management of this patient due to the risk of delayed and non-union of the femoral fracture. The risk of non-union in patients taking NSAIDs is 10 times higher than those not taking it in one study [3]. The inhibitory effect on osteoblasts is the main cause of the increased risk of delayed and non-union [3].

Radiation therapy: As with NSAIDs, prophylactic radiotherapy is used in patients undergoing total hip arthroplasty to prevent HO in high risk patients [4]. Nevertheless, very few literatures are available to support the usage of irradiation in patients with femoral fracture.

\section{Treatment}

Surgical resection: In symptomatic patients, mainstay of HO management is surgical resection with post-operative NSAIDs and/or radiotherapy to prevent recurrence [4]. When HO is fully ossified, surgical resection is recommended [4]. Serial radiograph of this patient show a lesion with clear margins and well-defined trabeculations indicating that the $\mathrm{HO}$ is mature. We have offered resection of the $\mathrm{HO}$ but this patient is not keen. By resecting $\mathrm{HO}$ when it is mature, it reduces complications such as haemorrhages and post-operative recurrence [4]. In our patient, conservative management by undergoing physiotherapy helps eliminate the pain. Hence, there is a role of conservative management if the patient refuses to undergo surgical resection.

Biphosphonates therapy: First generation bisphosphonates, analogues of inorganic pyrophosphate, are able to inhibit the transformation of amorphous calcium phosphate into crystalline hydroxyapatite, resulting in the inhibition of bone matrix mineralization [8]. Early initiation (within 3-6 weeks of diagnosis) of biphosphanates such as etidronate has been shown to halt the progression of the HO once the diagnosis is made [8]. The treatment is most effective when the nuclear bone scan is positive, the radiographs are normal [8]. Biphosphanates are not as effective once it is shown on plain radiographs [8]. On top of that, first generation bisphosphonates such as etidronate has been shown to be poorly tolerated by the patients due to the various adverse effects of taking the medications, rendering it a suboptimal choice to treatment of HO. Newer generation of nitrogen-containing biphosphanates such as pamidronate and alendronate are more potent and better tolerated by the patients has been used to treat HO with promising outcomes (Figure $1 \& 2$ ). 


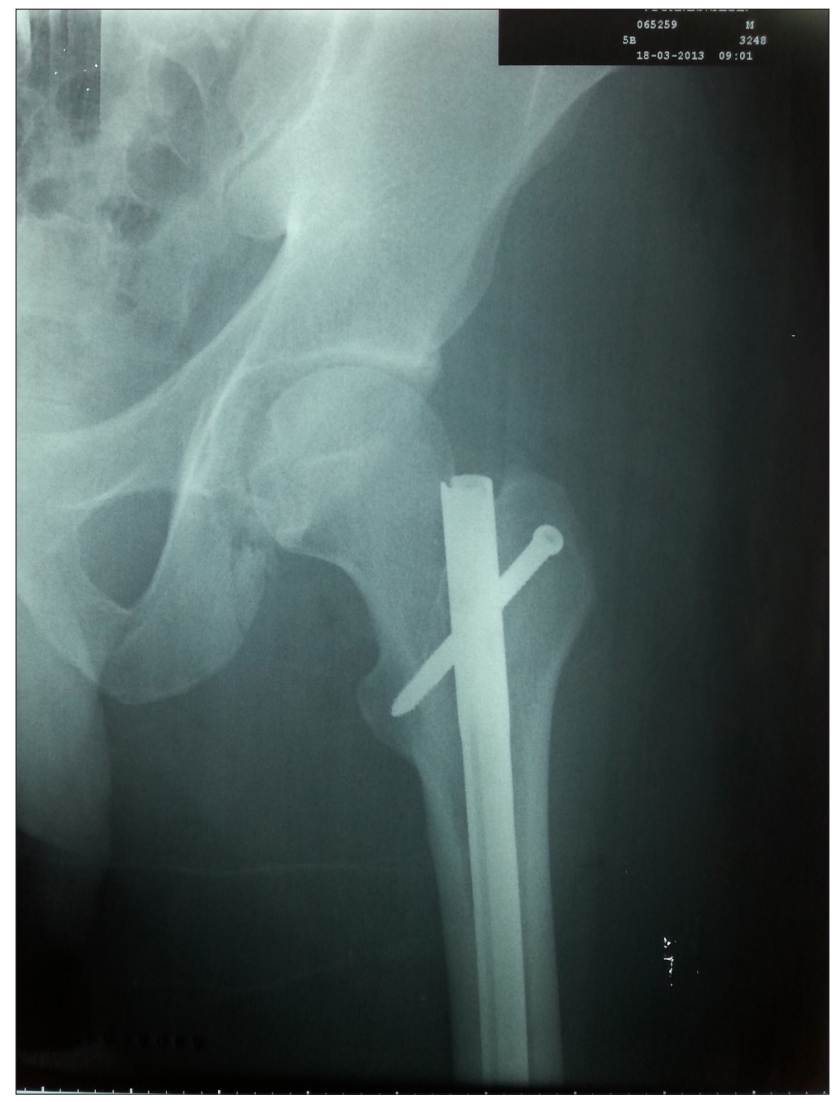

Figure 1: The radiograph of the left hip two days post reamed intramedullary nail.

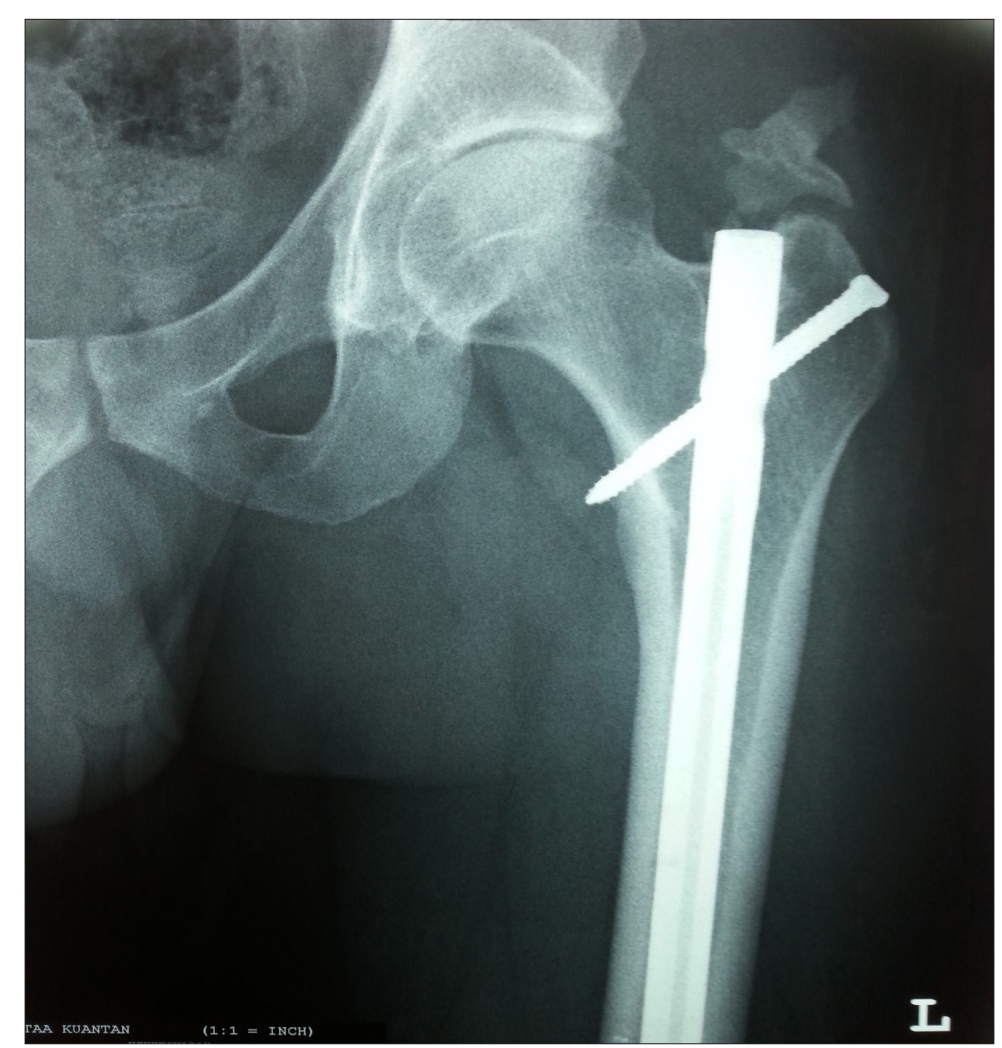

Figure 2: The radiograph of the left hip 13 months posts reamed intramedullary nail. There is a well-demarcated lesion superior to the left greater trochanter indicating heterotopic ossification at the left hip (arrow). 


\section{Conclusion}

This case highlights a common butoften neglected post traumatic complication of the hip. There is still lack of understanding of the exact pathophysiology of $\mathrm{HO}$ formation in patients undergoing IMN hence there is no consensus on the prevention and management of HO. Further randomized control studies are needed to better understand this disease for appropriate management.

\section{References}

1. Dodenhoff RM, Dainton JN, Hutchins PM (1997) Proximal thigh pain after femoral nailing. Causes and treatment. J Bone Joint Surg (Br) 79(5): 738-741.

2. Sergiu B, Cyril M, Mark-Hammerberg E, David JH, Philip FS (2013) Heterotopic ossification in the reaming tract of a percutaneous antegrade femoral nail: a case report. Journal of Medical Case Reports 7: 90.

3. Giannoudis PV, MacDonald DA, Matthews SJ, Smith RM, Furlong AJ, et al. (2000) Nonunion of the femoral diaphysis. The influence of reaming and non-steroidal anti-inflammatory drugs. J Bone Joint Surg (Br) 82(5): 655-658.
4. Dia Shebab, Abdelhamid HE, David CB (2002) Heterotopic ossification. J Nucl Med 43(3): 346-353.

5. Luc Vanden-Bossche, Guy V (2005) Heterotopic ossification: A review. J Rehabil Med 37(3): 129-136.

6. Legosz P, Otworowski M, Sibilska A, Starszak K, Kotrych D, et al. (2019) Heterotopic ossification: A challenging complications of total hip arthroplasty: risk factors, diagnosis, prophylaxis, and treatment. Bio Med Research International, pp. 1- 8.

7. Brumback RJ, Wells JD, Lakatos R, Poka A, Bathon GH, et al. (1990) Heterotopic ossification about the hip after intramedullary nailing for fracture of the femur. J Bone Joint Surg Am 72(7): 1067-1073.

8. Teasell RW, Mehta S, Aubut JL, Ashe MC, Sequeira K, et al. (2010) A systematic review of therapeutic interventions for heterotopic ossification following spinal cord injury. Spinal Cord 48(7): 512-521.

For possible submissions Click below: 